\title{
Swept volumes: void and boundary identification
}

\author{
Karim Abdel-Malek ${ }^{1 *}$, Harn-Jou Yeh ${ }^{2}$ and Saeb Othman ${ }^{3}$
}

A general formulation for determining complex sweeps comprising a multiple of parameters has been presented by the authors in recent work. This paper investigates the boundaries to swept volumes, and in specific, addresses the problem of determination of voids in the volume. The determination of voids has become of major concern in CAD software, where the accurate calculation of the swept volume is used in computing solid properties such as mass and moments of inertia. A mathematical formulation based on the concept of a normal acceleration function on singular surfaces is introduced. Criteria are derived regarding the identification of a boundary from the definiteness properties of the normal acceleration function. Numerical examples are illustrated in detail and represent the first treatment of void identification in complex sweeps. (C) 1999 Elsevier Science Ltd. All rights reserved

Keywords: swept volume, void detection, boundary identification, sweep Jacobian

\section{INTRODUCTION}

Formulations for sweeps of entities with only three parameters such as the sweep of a surface along a curve are well established. However, the need for modeling complex parts has necessitated different approaches, one of which is the multiple sweeping of entities. Using this method, the resulting shape of one sweep operation is swept again and again until the final desired shape is obtained. This type of design process yields complex solid bodies that are represented by the sweep equation of many parameters and presents a versatile tool for the designer.

Moreover, identification of boundaries and voids is of vital importance to $\mathrm{CAD}$ environments where the accurate computation of the swept volume is used in estimating the mass and inertia properties of a solid. In the collision

${ }^{*}$ Corresponding author. Tel: 001319335 5679; fax: 001319335 5669; e-mail: amalek@icaen.uiowa.edu

${ }^{1}$ Department of Mechanical Engineering and Center for Computer-Aided Design, The University of Iowa, Iowa City, IA 52242, USA

${ }^{2}$ Microtek Inc., Taiwan

${ }^{3}$ National Advanced Driving Simulator (NADS), The University of Iowa, Oakdale, IA 52240, USA

Paper Received: 27 April 1998. Revised: 26 July 1998. Accepted: 3 August 1998 detection of robotic manipulators, it is equally important to define the exact envelope of each moving link, especially those trajectories that are due to a multiple of parameters.

This paper presents a new method for the identification of boundary curves and surface patches of a swept volume of any parameter. Although the problem has been treated to some extent in the past, the proposed method is based on mathematical criteria derived from acceleration analysis adapted from kinematics. A broadly applicable formulation and its accompanying algorithm are presented.

The first introduction of a rigorous formulation for calculating critical curves from a moving solid was introduced by Wang and Wang ${ }^{22}$. Sweeps involving a larger number of parameters have become necessary in CAD systems where subsequent sweeps yield more complex shapes. Swept volumes of higher order parameters, based on Jacobian rank deficiency conditions, have been presented by the first two authors ${ }^{1}$ and was shown to handle multiple sweeps resulting in more than three parameters.

Work presented by $\mathrm{Hu}$ and Ling ${ }^{12,13}$ introduces the swept volume generated by natural quadratic surfaces. This work uses instantaneous screw axis motion used in kinematics to determine the sweep of a surface implicitly described but does not address the existence or the identification of voids in the workspace.

Also using the envelope theory, Martin and Stephenson ${ }^{18}$ evaluated the swept volume of a three-dimensional object moving along an arbitrary path. Although they addressed self intersection of the envelope, they did not address the identification of boundaries to voids.

Kieffer and Litvin ${ }^{15}$ developed an algorithm for the determination of all surfaces and edges, generated by the sweep, that may (but not necessarily) bound the swept volume. Computations of volumes swept by polyhedral objects in order to compute viewpoints for monitoring objects and features in an active robot work-cell is reported by Abrams and Allen ${ }^{2}$. Another type of mathematical approach is defined by Schroeder and Lorensen ${ }^{20}$ as the minimization of the sum of distances from the moving object (a polygon). Algebraic methods for implicit swept solids were presented by Ganter et al. ${ }^{11}$ while other methods were also reported ${ }^{3,7,8,16}$. Sweep differential techniques were efficiently implemented to swept volume analysis, manipulator workspace, and modeling ${ }^{4-6,17,23}$. Using sweep differential techniques, the authors have also addressed the implementation of expansions and contractions in lieu of rotational matrices. Although not directly addressed by the authors in 
any of the papers, it seems that the sweep differential technique is capable of identifying voids in the volume, as evidenced in one of their works ${ }^{23}$.

Swept volume implementation in NC verification was treated by several authors ${ }^{10,14,19,21}$. Identification of voids in swept volumes was only addressed using sweep differential techniques.

We shall first present a brief review of the formulation for the determination of closed form surface equations that exist in the volume. The formulation takes into consideration inequality constraints imposed on the sweep parameters. The concept of a normal acceleration on a singular surface will then be developed. An additional criterion for the normal function at the boundary of an entity will then be presented, followed by illustrative examples.

\section{BACKGROUND}

Define a geometric entity parametrized in terms of one or more variables as a $(3 \times 1)$ vector given by $\Gamma(\mathbf{w})$, where $\mathbf{u}=\left[u_{1}, \ldots, u_{n}\right]^{\mathrm{T}}$, i.e. the geometric entity can be a curve, a surface, or an entity in a higher dimensional space. The entity $\Gamma(\mathbf{w})$ will be swept along a second entity, $\Psi(\mathbf{v})$, where $\mathbf{v}=\left[v_{1}, \ldots, v_{m}\right]^{\mathrm{T}}$. The set generated by this sweep is defined as:

$$
\Phi(\mathbf{q})=\mathbf{R}(\mathbf{v}) \Gamma(\mathbf{w})+\Psi(\mathbf{v})
$$

where $\Phi(\mathbf{q}) \in \mathcal{R}^{3}$ and $\mathbf{q}$ is the extended vector defined by:

$$
\mathbf{q}=\left[q_{1}, \ldots, q_{n+m}\right]^{\mathrm{T}}=\left[w_{1}, \ldots, w_{n}, v_{1}, \ldots, v_{m}\right]^{\mathrm{T}}
$$

and $\mathbf{R}(\mathbf{v})$ is the $(3 \times 3)$ rotation matrix defining the orientation of the swept entity. In fact, $\Phi(\mathbf{q})$ characterizes the set of points belonging to the swept volume (a position vector). Singular surfaces/curves (so-called hyperentities) ${ }^{1,18}$ comprising the swept volume were analytically determined by applying rank deficiency conditions to the sweep Jacobian. For the set, $\Theta(\mathbf{q})$, the dimension of the null space of the Jacobian $\Phi_{\mathbf{q}}=\left[\partial \Theta_{i} / \partial q_{j}\right]$ is associated with singular behavior characterized by the singular sets $\mathbf{p}^{(i)}$. The sets $\mathbf{p}^{(i)}$ are constant parameters that are determined from the Jacobian rank deficiency condition as first introduced in Abdel-Malek and Yeh ${ }^{1}$.

It was shown that substituting $\mathbf{p}^{(i)}$ into $\Phi(\mathbf{q})$ yields hyperentities parametrized by $\xi^{(i)}\left(\mathbf{u}^{(i)}\right)$ where $\mathbf{u}^{(i)}$ is the new vector of generalized coordinates such that:

$$
\Phi\left(\mathbf{u}^{(i)}, \mathbf{p}^{(i)}\right)=\xi^{(i)}\left(\mathbf{u}^{(i)}\right)
$$

where $\mathbf{q}^{(i)^{\mathrm{T}}}=\left[\mathbf{u}^{(i)^{\mathrm{T}}} \mathbf{p}^{(i)^{\mathrm{T}}}\right]$ and $\mathbf{u}^{(i)} \cap \mathbf{p}^{(i)}=\phi$. A convenient parametrization of constraints imposed on $\mathbf{q}$ was also presented such that the inequality constraint $q_{i}^{\mathrm{L}} \leq q_{i} \leq q_{i}^{\mathrm{U}}$ is parametrized as:

$$
q_{i}\left(\lambda_{i}\right)=\frac{\left(q_{i}^{\mathrm{U}}+q_{i}^{\mathrm{L}}\right)}{2}+\frac{\left(q_{i}^{\mathrm{U}}-q_{i}^{\mathrm{L}}\right)}{2} \sin \lambda_{i}
$$

where new parameters were introduced such that $\mathbf{q}=\mathbf{q}(\mathbf{s})$, where $\mathbf{s}=\left[\lambda_{1}, \lambda_{2}, \ldots, \lambda_{n}\right]^{\mathrm{T}}$ and $\Phi=\Phi[\mathbf{q}(\mathbf{s})]$ is now a function of the new variables (usually called slack variables in the field of optimization).

It was also shown that the basis of the null space of $\Phi_{\mathrm{q}}^{\mathrm{T}}$ is a vector normal to hyperentities in the workspace, i.e. a vector $\mathbf{n}_{0}$ evaluated at $\mathbf{q}_{0}$ satisfies:

$$
\Phi_{\mathbf{q}}^{\mathrm{T}} \mathbf{n}_{0}=\mathbf{0}
$$

where $\mathbf{n}_{0}=\left[\begin{array}{lll}\gamma_{1} & \gamma_{2} & \gamma_{3}\end{array}\right]^{\mathrm{T}}$, then $\mathbf{n}_{0}$ is a vector normal to hyperentities at $\mathbf{q}_{0}$. A perturbation method was used to identify the boundary to the swept volume. Some difficulties were encountered in performing the perturbation since a solution to a set of non-linear equations had to be found for every perturbed point, i.e. the identification of the boundary remained problematic. Voids in the swept volume were not addressed.

\section{CONCEPT OF NORMAL ACCELERATION}

The main objective of this section is to derive a formulation for identifying the boundary (whether an exterior boundary or a boundary to a void). The concept of normal acceleration adapted from kinematics will be implemented to a fictitious point on a hyperentity. If surfaces are considered as parametrized with respect to time, then this point would be a position triplet at any instant of time.

The rationale for using a definition of normal acceleration will be first addressed. A point, $P$, on a smooth hyperentity, $H$, assumes motion, normal to the surface in either direction, depending on the difference between the component of acceleration normal to the surface and the component of acceleration along the principal normal, such that:

$$
\eta_{P}=\eta_{P}(H)=a_{\mathrm{n}}-\frac{v_{\mathrm{t}}^{2}}{\rho_{0}}
$$

where $v_{\mathrm{t}}$ is the tangential velocity, $a_{\mathrm{n}}$ is the component of acceleration normal to the hyperentity, and $1 / \rho_{0}$ is the normal curvature of the hypersurface (Figure 1$)$ with respect to the tangent direction of $v_{\mathrm{t}}\left(\rho_{0}\right.$ is the radius of curvature). At a boundary, the quantity in eqn (6) will only indicate a motion towards satisfying $\Phi$ and will indicate no possible motion towards the exterior of the swept volume.

The point will have no acceleration if the quantity computes to null. It will be shown that this quantity will indeed identify a boundary and a void since, at those hyperentities, eqn (6) will indicate no motion into the void, or towards the exterior of the swept volume since the void is not a subset of the set $\Phi[\mathbf{q}(\mathbf{s})]$. Part of a hypersurface enveloping a void in the volume must admit motion only towards the exterior of that void.

This concept will be generalized in the discussion that follows and will be shown to yield a quadratic form. It will also be shown that these boundaries do not admit motion regardless of the values of velocity or acceleration at the point of interest, but will only depend on the definiteness properties of the quadratic form.

In order to apply eqn (6) to hyperentities, it is first necessary to define the first and second fundamental

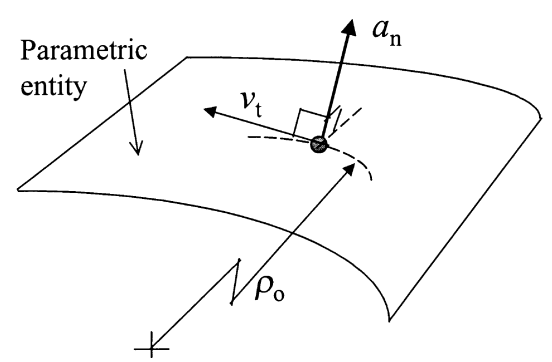

Figure 1 Concept of normal acceleration 
form $s^{9}$ denoted by $\mathbf{I}_{p}$ and $\mathbf{I I}_{p}$, respectively, of a parametric geometric entity $\xi(\mathbf{u})$, where $\mathbf{u}=\left[\begin{array}{ll}u & v\end{array}\right]^{\mathrm{T}}$. The first fundamental form is defined as:

$$
\mathbf{I}_{p} \equiv \delta \mathbf{u}^{\mathrm{T}} \xi_{\mathrm{u}}^{\mathrm{T}} \xi_{\mathrm{u}} \delta \mathbf{u}
$$

where $\xi_{\mathrm{u}}=\partial \xi / \partial \mathbf{u}$ and the second fundamental form is defined as:

$$
\mathbf{I I}_{p} \equiv \delta \mathbf{u}^{\mathrm{T}}\left[\mathbf{n}^{\mathrm{T}} \xi\right]_{\mathrm{uu}} \delta \mathbf{u}
$$

or expanded as:

$$
\mathbf{I I}_{p}=\mathbf{n}^{\mathrm{T}} \xi_{u u} \mathrm{~d} u^{2}+2 \mathbf{n}^{\mathrm{T}} \xi_{u v} \mathrm{~d} u \mathrm{~d} v+\mathbf{n}^{\mathrm{T}} \xi_{v v} \mathrm{~d} v^{2}
$$

where $\mathbf{n}$ is the normal vector to the hypersurface and $\xi_{u v}=\partial^{2} \xi / \partial u \partial v$. The normal curvature, $K_{0}$, of a parametric surface at a configuration, $\mathbf{q}_{0}$, in the direction of $\mathrm{d} u / \mathrm{d} v$, can then be defined as the ratio:

$$
K_{0}=\frac{1}{\rho_{0}}=\frac{\mathbf{I}_{p}}{\mathbf{I}_{p}}
$$

Define the time-modified first and second fundamental forms as:

$$
\begin{aligned}
& \mathbf{I}_{p}^{\prime} \equiv \dot{\mathbf{u}}^{\mathrm{T}} \xi_{\mathrm{u}}^{\mathrm{T}} \xi_{\mathrm{u}} \dot{\mathbf{u}} \\
& \mathbf{I I}_{p}^{\prime} \equiv \dot{\mathbf{u}}^{\mathrm{T}}\left[\mathbf{n}^{\mathrm{T}} \xi\right]_{\mathrm{uu}} \dot{\mathbf{u}}
\end{aligned}
$$

such that the normal curvature can still be defined as:

$$
K_{0}=\frac{1}{\rho_{0}}=\frac{\mathbf{I I}_{p}}{\mathbf{I}_{p}}=\frac{\mathbf{I}_{p}^{\prime}}{\mathbf{I}_{p}^{\prime}}
$$

In general, the tangential velocity in terms of $\xi$ or $\Phi$ at any point on the surface is:

$$
\mathbf{v}_{\mathrm{t}}=\xi_{\mathrm{u}} \dot{\mathbf{u}}=\Phi_{\mathrm{q}} \dot{\mathbf{q}}
$$

where $\dot{\mathbf{u}}=\mathbf{u}_{\mathrm{s}} \dot{\mathbf{s}}$ and $\dot{\mathbf{q}}=\mathbf{q}_{\mathrm{s}} \dot{\mathbf{s}}$. The squared norm of the velocity is:

$$
\left\|\mathbf{v}_{\mathrm{t}}\right\|^{2}=\mathbf{v}_{\mathrm{t}}^{\mathrm{T}} \mathbf{v}_{\mathrm{t}}=\dot{\mathbf{u}}^{\mathrm{T}} \xi_{\mathrm{u}}^{\mathrm{T}} \xi_{\mathrm{u}} \dot{\mathbf{u}}
$$

which is equal to the modified first fundamental form $\mathbf{I}_{p}^{\prime}$ of eqn (11). Therefore, $\mathbf{I}_{p}$ can be written as:

$$
\mathbf{I}_{p}^{\prime}=\left|v_{\mathrm{t}}\right|^{2}
$$

The objective is to derive an expression for $\eta$ for any hyperentity at a specified point.

\section{An expression for $\mathbf{I I}_{p}^{\prime}$}

Substituting for $1 / \rho_{0}$ into $\eta$ and using eqn (16) yields:

$$
\eta=a_{\mathrm{n}}-\left.v_{\mathrm{t}}\right|^{2} \frac{\mathbf{I}_{p}^{\prime}}{\mathbf{I}_{p}^{\prime}}=a_{\mathrm{n}}-\mathbf{I I}_{p}^{\prime}
$$

Since $a_{\mathrm{n}}$ is in terms of $\mathbf{q}$ and to express $\mathbf{I I}_{p}^{\prime}$ in terms of $\dot{\mathbf{q}}$, the velocity vector $(\dot{\mathbf{u}})$ on a hypersurface is extracted from eqn (14). Since $\xi_{\mathrm{u}}$ is not square, a generalized inverse can be written such that:

$$
\dot{\mathbf{u}}=\mathbf{B} \Phi_{\mathrm{q}} \dot{\mathbf{q}}
$$

or

$$
\dot{\mathbf{u}}=\mathbf{B} \Phi_{\mathrm{q}} \mathbf{q}_{\mathrm{s}} \dot{\mathbf{s}}
$$

where $\mathbf{B}$ is the generalized inverse defined by $\mathbf{B}=\left[\mathbf{E} \xi_{\mathrm{u}}\right]^{-1} \mathbf{E}$ and:

$$
\mathbf{E}=\left[\begin{array}{lll}
1 & 0 & 0 \\
0 & 1 & 0
\end{array}\right] \text { if the first and second rows of } \xi_{\mathrm{u}}
$$

are independent

$$
\mathbf{E}=\left[\begin{array}{lll}
1 & 0 & 0 \\
0 & 0 & 1
\end{array}\right] \text { if the first and third rows of } \xi_{\mathrm{u}}
$$

are independent

$$
\begin{aligned}
\mathbf{E}= & {\left[\begin{array}{lll}
0 & 1 & 0 \\
0 & 0 & 1
\end{array}\right] \text { if the second and third rows of } \xi_{\mathrm{u}} } \\
& \text { are independent }
\end{aligned}
$$

Substituting the expression for $\dot{\mathbf{u}}$ into eqn (12) yields:

$$
\mathbf{I}_{p}^{\prime}=\dot{\mathbf{u}}^{\mathrm{T}}\left[\mathbf{n}^{\mathrm{T}} \xi\right]_{\mathrm{uu}} \dot{\mathbf{u}}=\dot{\mathbf{s}}^{\mathrm{T}} \mathbf{q}_{\mathrm{s}} \Phi_{\mathrm{q}} \mathbf{B}^{\mathrm{T}}\left[\mathbf{n}^{\mathrm{T}} \xi\right]_{\mathrm{uu}} \mathbf{B} \Phi_{\mathrm{q}} \mathbf{q}_{\mathrm{s}} \dot{\mathbf{s}}
$$

which is a general expression for $\mathbf{I I}_{p}^{\prime}$.

\section{An expression for $a_{\mathrm{n}}$}

An expression for the velocity at any point in the swept volume is obtained by differentiating $\Phi(\mathbf{s})$ with respect to time as:

$$
\dot{\Phi}=\Phi_{\mathrm{q}} \mathbf{q}_{\mathrm{s}} \dot{\mathbf{s}}
$$

On a hypersurface, the term $\left.\Phi_{\mathrm{q}} \mathbf{q}_{\mathrm{s}}\right|_{\mathrm{q}_{0}, \mathrm{~s}_{0}}$ is rank-deficient. Therefore, the rows of $\Phi_{\mathrm{q}} \mathbf{q}_{\mathrm{s}}$ are dependent and there exists a set of constants, $\mathbf{n}_{0}=\left[\begin{array}{lll}\gamma_{1} & \gamma_{2} & \gamma_{3}\end{array}\right]^{\mathrm{T}}$ that satisfy:

$$
\left[\Phi_{\mathrm{q}} \mathbf{q}_{\mathrm{s}}\right]^{\mathrm{T}} \mathbf{n}_{0}=\mathbf{0}
$$

where $\mathbf{n}_{0}$ is the normal vector to a hypersurface at $\mathbf{q}_{0}$ [identical to eqn (5)].

Differentiating eqn (21) again in indicial form, the acceleration at any point is:

$$
\ddot{\Phi}_{i}=\frac{\mathrm{d}}{\mathrm{d} t}\left[\frac{\mathrm{d} \Phi_{i}}{\mathrm{~d} q_{j}}\right] \frac{\mathrm{d} q_{j}}{\mathrm{~d} s_{k}} \dot{s}_{k}+\left[\frac{\mathrm{d} \Phi_{i}}{\mathrm{~d} q_{j}}\right] \frac{\mathrm{d}}{\mathrm{d} t}\left(\frac{\mathrm{d} q_{j}}{\mathrm{~d} s_{k}}\right) \dot{s}_{k}+\left[\frac{\mathrm{d} \Phi_{i}}{\mathrm{~d} q_{j}}\right] \frac{\mathrm{d} q_{j}}{\mathrm{~d} s_{k}} \ddot{s}_{k}
$$

Expanding the derivatives and collecting similar terms yields:

$$
\ddot{\Phi}_{i}=\dot{s}_{n}\left\{\frac{\mathrm{d} q_{j}}{\mathrm{~d} s_{n}}\left[\frac{\mathrm{d}^{2} \Phi_{i}}{\mathrm{~d} q_{m} \mathrm{~d} q_{j}}\right] \frac{\mathrm{d} q_{j}}{\mathrm{~d} s_{k}}+\frac{\mathrm{d} \Phi_{i}}{\mathrm{~d} q_{j}} \frac{\mathrm{d}^{2} q_{j}}{\mathrm{~d} s_{n} \mathrm{~d} s_{k}}\right\} \dot{s}_{k}+\left[\frac{\mathrm{d} \Phi_{i}}{\mathrm{~d} q_{j}} \frac{\mathrm{d} q_{j}}{\mathrm{~d} s_{k}}\right] \ddot{s}_{k}
$$

In matrix form, eqn (24) is written as:

$$
\ddot{\Phi}=\dot{\mathbf{s}}\left\{\mathbf{q}_{\mathrm{s}}^{\mathrm{T}} \Phi_{\mathrm{qq}} \mathbf{q}_{\mathrm{s}}+\sum_{i=1}^{n+m} \frac{\mathrm{d} \Phi}{\mathrm{d} q_{i}} \cdot\left[q_{i}\right]_{\mathrm{ss}}\right\} \dot{\mathbf{s}}+\left[\Phi_{\mathrm{q}} \mathbf{q}_{\mathrm{s}}\right] \ddot{\mathbf{s}}
$$

To obtain the normal acceleration, $\dot{\Phi}$ is projected onto the normal $\mathbf{n}_{0}$. Multiplying both sides of eqn (25) by the vector $\mathbf{n}_{0}^{\mathrm{T}}$ eliminates the last term of the right hand side [definition of the normal as the basis of the null space in eqn (22)]. 
The component of the normal acceleration is then:

$$
a_{\mathrm{n}}=\mathbf{n}_{0}^{\mathrm{T}} \ddot{\Phi}=\dot{\mathbf{s}}^{\mathrm{T}} \mathbf{H}^{*} \dot{\mathbf{s}}
$$

where

$$
\mathbf{H}^{*}\left(\mathbf{q}_{0}, \mathbf{s}_{0}\right)=\mathbf{q}_{\mathrm{s}}^{\mathrm{T}}\left[\mathbf{n}_{0}^{\mathrm{T}} \Phi\right]_{\mathrm{qq}} \mathbf{q}_{\mathrm{s}}+\sum_{i=1}^{n} \frac{\mathrm{d}\left(\mathbf{n}_{0}^{\mathrm{T}} \Phi\right)}{\mathrm{d} q_{i}} \cdot\left[q_{i}\right]_{\mathrm{ss}}
$$

\section{A form for $\eta$}

In order to obtain an expression for $\eta$, substitute for $a_{\mathrm{n}}$ [from eqn (26)] and for $\mathbf{I I}_{p}^{\prime}$ [from eqn (20)] into eqn (17), such that:

$$
\eta=a_{\mathrm{n}}-\mathbf{I I}_{p}^{\prime}=\dot{\mathbf{s}}^{\mathrm{T}} \mathbf{Q}^{*} \dot{\mathbf{s}}
$$

where

$$
\mathbf{Q}^{*}=\mathbf{H}^{*}-\mathbf{q}_{\mathrm{s}}^{\mathrm{T}} \boldsymbol{\Phi}_{\mathrm{q}}^{\mathrm{T}} \mathbf{B}^{\mathrm{T}}\left[\mathbf{n}^{\mathrm{T}} \xi\right]_{\mathrm{uu}} \mathbf{B} \Phi_{\mathrm{q}} \mathbf{q}_{\mathrm{s}}
$$

where $\mathbf{Q}^{*}$ is a quadratic form. Definiteness properties of the quadraticform of eqn (28) determines whether motion can occur normal to a hypersurface in the direction of $\mathbf{n}^{\mathrm{T}}$ at a specified point (i.e. independent of the value of $\dot{\mathbf{s}}$ ). This form will indicate if a region on a hypersurface is a boundary of the swept volume, or is a boundary to a void. If $\mathbf{Q}^{*}$ is indefinite, the hyperentity admits motion along either normal direction and is not a boundary to the swept volume.

When $q_{i}$ is at a limit, i.e. $q_{i}^{\mathrm{L}}$ or $q_{i}^{\mathrm{U}}$, the quadratic form characterized by $\mathbf{Q}^{*}$ fails because $\dot{q}_{i}=\left[q_{i}^{\max }-q_{i}^{\min } / 2\right] \cos \lambda_{i}=0$ at an upper or lower bound since, at the boundary of a surface, $\lambda_{i}=-\pi / 2$ or $\pi / 2$. Therefore, some terms in $\mathbf{Q}^{*}$ are lost. A supplementary criterion is necessary for the boundary.

\section{Hyperentities with $q_{i}=q_{i}^{\text {limit }}$}

In the case of a parameter at its limit, eqn (28) yields a semidefinite quadratic form. For this case, we propose the projection of a variational movement $\delta \Phi=\Phi_{q_{i}} \delta q_{i}$ due to $\delta q_{i}$ onto the normal direction, $\mathbf{n}$, such that the normal component is given by:

$$
\sigma=\mathbf{n}^{\mathrm{T}} \Phi_{q_{i}} \delta q_{i}
$$

where $\delta q_{i}$ is a specified magnitude of \pm 1 as follows:

$$
\delta q_{i}=\left\{\begin{array}{l}
+1 \text { if } q_{i} \text { is at lower bound } \\
-1 \text { if } q_{i} \text { is at upper bound }
\end{array}\right.
$$

Positive values of $\sigma$ in eqn (30) indicate that the hyperentity can admit movement in the positive direction of $\mathbf{n}$. The criterion characterized by eqn (30) is used to supplement the criteria of the definiteness properties of $\mathbf{Q}^{*}$ to determine the boundary to voids inside the swept volume. The criteria are summarized as follows.

\section{VOID AND BOUNDARY IDENTIFICATION CRITERIA}

(1) If $\mathbf{Q}^{*}$ is indefinite (has both positive and negative eigenvalues), the hyperentity admits motion for each parameter, $q_{i}$, which is at its limit, and is not a boundary.
(2) If $\mathbf{Q}^{*}$ is either positive or negative semi-definite, an additional criterion must be evaluated. The value $\sigma$ in eqn (30) must be computed and:

- If $\sigma$ has a different sign than the nonzero eigenvalues of $\mathbf{Q}^{*}$, the hyperentity admits motion along its normal.

- If $\sigma$ has the same sign as the nonzero eigenvalues of $\mathbf{Q}^{*}$, the hyperentity does not admit motion along its normal. The direction, along which the hyperentity may admit motion, either in the positive or negative direction of $\mathbf{n}$, will be in the same sense of the sign of $\sigma$.

The geometric significance of the indefinite form is best perceived if explained in terms of the position of a point moving on the geometric entity. An indefinite quadratic form indicates that motion of this point in both normal directions is possible. Since motion is possible, then both sides of that surface (or curve) belong to the swept volume and hence this region is not a boundary. If the quadratic form is either positive or negative semi-definite, then the supplementary criterion is applied. In this case, one of the sides is definitely not a boundary, however, the other side of the surface may admit motion of that point into the direction of the swept volume. The computational algorithm for detecting whether a surface region admits normal motion is shown in Appendix A.

\section{EXAMPLES}

\section{A three-parameter 2D example}

Consider the simple example of the consecutive sweep of a curve. The curve will first be swept about an axis, and the resulting shape swept again about a different axis. Three sets of boundary curves will be identified using the proposed method. This example is identical to the determination of the workspace of a planar three degrees of freedom manipulator.

The circular curve shown in Figure $2 a$ is defined by $\Gamma(\mathbf{w})=\left[\begin{array}{lll}\cos q_{3} & \sin q_{3} & 0\end{array}\right]^{\mathrm{T}}$ with respect to the $Z_{1}$ axis and constrained by $-\pi / 2 \leq q_{3} \leq \pi / 2$. This curve is to be swept about the axis $Z_{1}$; its projection is also shown in Figure $2 a$. The rotation matrix for this sweep is:

$$
\mathbf{R}\left(q_{2}\right)=\left[\begin{array}{ccc}
\cos q_{2} & -\sin q_{2} & 0 \\
\sin q_{2} & \cos q_{2} & 0 \\
0 & 0 & 1
\end{array}\right]
$$

where the rotation angle is constrained by $-\pi \leq q_{2} \leq \pi / 6$ and the position vector from the axis to the center of the curve is $\Psi\left(q_{2}\right)=\left[\begin{array}{lll}3 \cos q_{2} & 3 \sin q_{2} & 0\end{array}\right]^{\mathrm{T}}$.

Therefore, the first sweep equation is:

$$
\begin{aligned}
& \mathbf{K}\left(q_{2}, q_{3}\right) \\
& =\left[\begin{array}{lll}
3 \cos q_{2}+\cos \left(q_{2}+q_{3}\right) & 3 \sin q_{2}+\sin \left(q_{2}+q_{3}\right) & 0
\end{array}\right]^{\mathrm{T}}
\end{aligned}
$$

This shape will now be swept again about a second axis about $Z_{2}$ (Figure $2 b$ ). The rotation matrix is:

$$
\mathbf{R}\left(q_{1}\right)=\left[\begin{array}{ccc}
\cos q_{1} & -\sin q_{1} & 0 \\
\sin q_{1} & \cos q_{1} & 0 \\
0 & 0 & 1
\end{array}\right]
$$




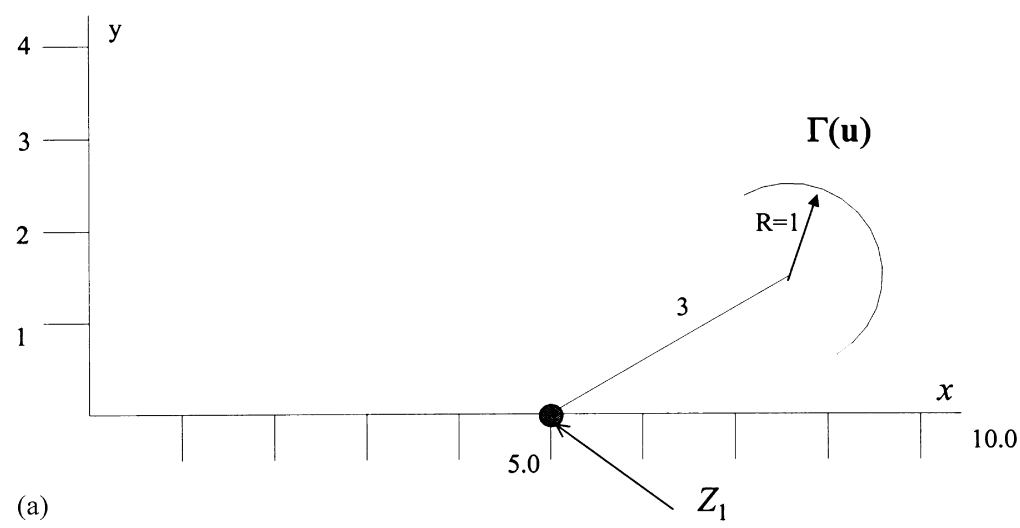

(a)

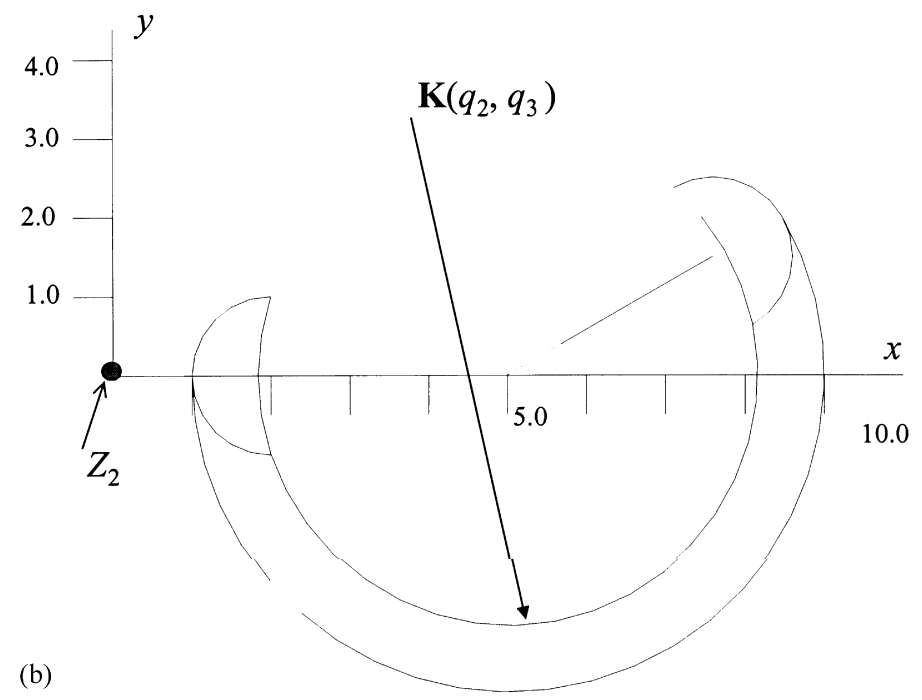

Figure 2 (a) A curve swept about $Z_{1}$. (b) The resultant shape swept about $Z_{2}$

with a position vector $\Psi\left(q_{1}\right)=\left[\begin{array}{lll}5 \cos q_{1} & 5 \sin q_{1} & 0\end{array}\right]^{\mathrm{T}}$ and a rotation angle $0 \leq \mathrm{q}_{1} \leq 330^{\circ}$. The new sweep equation is: $0, q_{2}=-\pi / 6, \quad \mathbf{p}^{(3)}=q_{1}=0, q_{3}=-\pi / 2, \quad \mathbf{p}^{(4)}=q_{1}=0$,
$q_{3}=\pi / 2, \quad \mathbf{p}^{(5)}=q_{2}=-\pi, q_{3}=-\pi / 2, \quad \mathbf{p}^{(6)}=q_{2}=-\pi$,

$$
\Phi(\mathbf{q})=\mathbf{R}\left(q_{1}\right) \mathbf{K}\left(q_{2}, q_{3}\right)+\Psi\left(q_{1}\right)=\left[\begin{array}{llll}
5 \cos q_{1}+3 \cos \left(q_{2}+q_{3}\right)+\cos \left(q_{1}+q_{2}+q_{3}\right) & 5 \sin q_{1}+3 \sin \left(q_{2}+q_{3}\right)+\sin \left(q_{1}+q_{2}+q_{3}\right) & 0
\end{array}\right]^{\mathrm{T}}
$$

The sweep Jacobian is computed as:

$$
\Phi_{\mathbf{q}}=\left[\begin{array}{ccc}
-5 \sin q_{1}-3 \sin \left(q_{1}+q_{2}\right)-\sin \left(q_{1}+q_{2}+q_{3}\right) & -3 \sin \left(q_{1}+q_{2}\right)-\sin \left(q_{1}+q_{2}+q_{3}\right) & -\sin \left(q_{1}+q_{2}+q_{3}\right) \\
5 \cos q_{1}+3 \cos \left(q_{1}+q_{2}\right)+\cos \left(q_{1}+q_{2}+q_{3}\right) & 3 \cos \left(q_{1}+q_{2}\right)+\cos \left(q_{1}+q_{2}+q_{3}\right) & \left.\cos \left(q_{1}\right]+q_{2}+q_{3}\right)
\end{array}\right]
$$

Using the criteria presented in Abdel-Malek and $\mathrm{Yeh}^{1}$, Jacobian rank-deficiency conditions resulting from the row rank deficiency of $\left[\Phi_{\mathrm{q}}\right]^{\mathrm{T}}$ necessitate the equating of the determinants of the three sub-Jacobians to zero, such that:

$$
\begin{aligned}
& \left|j_{1}\right|=3\left[3 \sin q_{2}+\sin \left(q_{2}+q_{3}\right)\right]=0 \\
& \left|j_{2}\right|=3 \sin q_{3}+5 \sin \left(q_{2}+q_{3}\right)=0 \\
& \left|j_{3}\right|=3 \sin q_{3}=0
\end{aligned}
$$

Solutions to the above three equations that satisfy the inequality constraints are $\mathbf{p}^{(19)}=q_{2}=0, q_{3}=0$ and $\mathbf{p}^{(17)}=q_{1}=0, q_{0}=0$ The reduced-order set yield $\mathbf{p}^{(13)}=$ $q_{2}=0.3217, q_{3}=-\pi / 2, \quad \mathbf{p}^{(14)}=q_{2}=0.3217, q_{3}=\pi / 2$, $\mathbf{p}^{(15)}=q_{2}=-\pi, q_{3}=0, \quad \mathbf{p}^{(16)}=q_{2}=\pi / 6, q_{3}=-0.3287$ and $\mathbf{p}^{(18)}=q_{1}=33 \pi / 18, q_{3}=0$. Constraint singular sets are computed as $\mathbf{p}^{(1)}=q_{1}=0, q_{2}=-\pi, \quad \mathbf{p}^{(2)}=q_{1}=$ $q_{3}=\pi / 2, \quad \mathbf{p}^{(7)}=q_{2}=\pi / 6, q_{3}=-\pi / 2, \quad \mathbf{p}^{(8)}=q_{2}=\pi / 6$, $q_{3}=\pi / 2, \quad \mathbf{p}^{(9)}=q_{1}=33 \pi / 18, q_{2}=-\pi, \quad \mathbf{p}^{(10)}=q_{1}=$ $33 \pi / 18, q_{2}=\pi / 6, \quad \mathbf{p}^{(11)}=q_{1}=33 \pi / 18, q_{3}=-\pi / 2 \quad$ and $\mathbf{p}^{(12)}=q_{1}=33 \pi / 18, q_{3}=\pi / 2$. Substituting these singular sets into eqn (33) yields parametric equations of curves (hypercurves) shown in Figure 3.

To determine any voids, it is necessary to evaluate these criteria for all curve segments. For example, consider a point $A_{1}$ on curve $\zeta^{(17)}(\mathbf{u})$, shown in Figure 4 , where $\mathbf{u}^{(17)}$

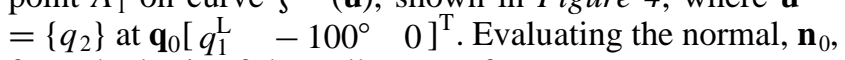
from the basis of the null space of:

$$
\left[\Phi_{\mathrm{q}} \mathbf{q}_{\lambda}\right]^{\mathrm{T}}=\left[\begin{array}{ccc}
0 & 7.0114 & 1.5469 \\
0 & -1.2363 & -0.2727
\end{array}\right]
$$

at $A_{1}$, yields the normal $\mathbf{n}_{0}=\left[\begin{array}{ll}-0.1736 & -0.9848\end{array}\right]^{\mathrm{T}}$. Since this is a $2 \mathrm{D}$ three-parameter example, the hypercurve is parametrized by a single variable, therefore, $\Phi_{q_{1}}$ is a ( $2 \times$ 


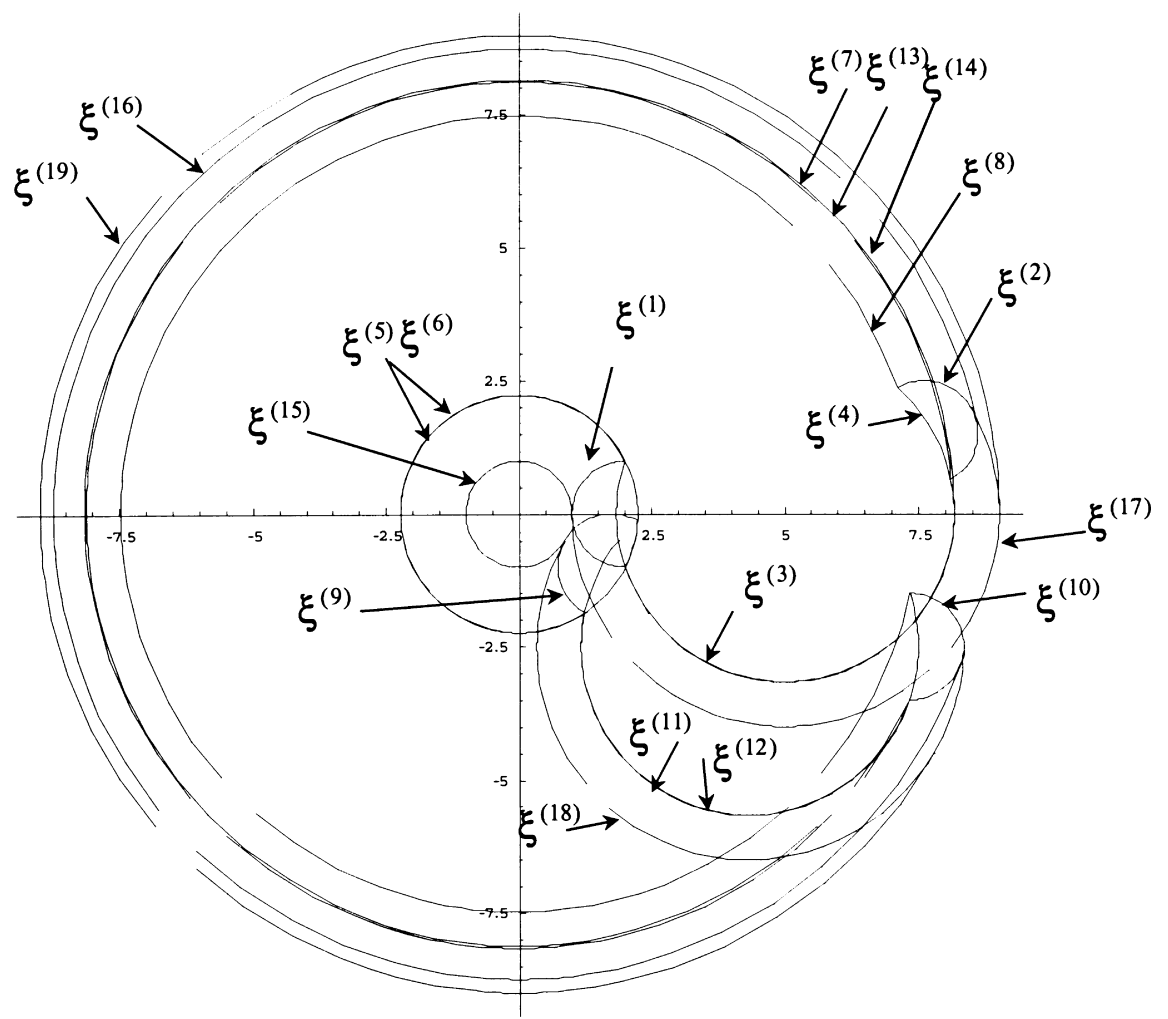

Figure 3 Hypercurves of Example 1 (see Figure 4 for void identification)

1) matrix. The matrix $\mathbf{E}$ is therefore, $\mathbf{E}=\left[\begin{array}{ll}1 & 0\end{array}\right]$ and

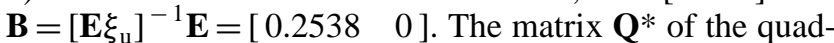
ratic form is evaluated as:

$$
\mathbf{Q}^{*}=\left[\begin{array}{ccc}
-14.1802 & 0 & 0 \\
0 & 0 & 0 \\
0 & 0 & -1.8505
\end{array}\right]
$$

The eigenvalues of $\mathbf{Q}^{*}$ are evaluated as $\{-14.1802$, $-1.8505,0\}$, which indicates a negative semi-definite quadratic form. Since $q_{1}$ is, at its lower bound $\left(q_{1}^{\mathrm{L}}\right)$, the additional value of $\sigma$ is evaluated with $\delta q_{2}=+1$. The value for $\sigma=\mathbf{n}^{\mathrm{T}} \mathbf{x}_{q_{2}} \delta q_{2}=-4.9240$. Since the sign of $\sigma$ is the same as that of the eigenvalues of $\mathbf{Q}^{*}$, it indicates that the surface $\xi^{(17)}$ at $A_{1}$ only admits movement into the opposite direction

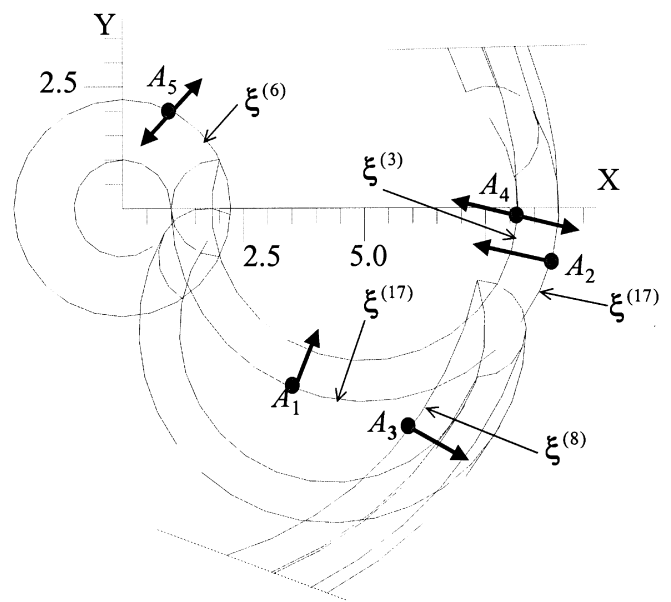

Figure 4 Part of the swept area showing curve segments of the normal $\mathbf{n}_{0}$ (an arrow shows the admissible direction in Figure 4). This indicates that this curve is a boundary.

Similarly, for the same curve at point $A_{2}$ at $\mathbf{q}_{0}=\left[\begin{array}{lll}q_{1}^{\mathrm{L}} & -10^{\circ} & 0\end{array}\right]^{\mathrm{T}}$, which is an exterior boundary, the normal is $\mathbf{n}=\left[\begin{array}{ll}-0.985 & 0.17\end{array}\right]^{\mathrm{T}}$ and the eigenvalues are computed as $\{2.5,1.8505,0\}$ and $\sigma=0.868$; the same sign as the eigenvalues. This indicates an admissible motion in that direction (but not the opposite direction, i.e. a boundary).

On curve $\xi^{(18)}$, at point $A_{3}$, (also shown in Figure 4) at $q_{0}=\left[\begin{array}{lll}3050 & q_{2}^{\mathrm{U}} & q_{3}^{\mathrm{U}}\end{array}\right]^{\mathrm{T}}$, the normal is $n_{0}=$ $\left[\begin{array}{ll}-0.8032 & 0.5957\end{array}\right]^{\mathrm{T}}$ and the eigenvalues are computed as $\{-2.8974,-1.5389,0\}$ and $\sigma=-1.5810$. On curve $\zeta^{(3)}$, at point $A_{4}$, at $\mathbf{q}_{0}=\left[\begin{array}{lll}q_{1}^{\mathrm{L}} & 0 & q_{3}^{\mathrm{L}}\end{array}\right]^{T}$, the normal is $\mathbf{n}_{0}=\left[\begin{array}{ll}-0.9487 & 0.3160\end{array}\right]^{\mathrm{T}}$ and the eigenvalues are computed as $\{4.5529,-1.4901,0\}$ which is an indefinite quadratic form, i.e. it does not represent a boundary.

Similarly, at point $A_{5}$, on curve $\zeta^{(6)}$, at $\mathbf{q}_{0}=\left[\begin{array}{lll}\pi / 2 & q_{2}^{\mathrm{L}} & q_{3}^{\mathrm{L}}\end{array}\right]^{\mathrm{T}}$, the normal is $\mathbf{n}_{0}=$ $\left[\begin{array}{ll}0.4472 & 0.8944\end{array}\right]^{\mathrm{T}}$ and the eigenvalues are computed as $\{-5.8849,4.0977,-1.4049\}$ which is an indefinite form, i.e. not a boundary.

It is evident that those segments of the hypercurves that do not admit motion belong to the boundary. For this example, there are three sets of boundary curves. The exterior boundary is characterized by the following segments: $\zeta^{(19)}\left(q_{1}\right): 0 \leq q_{1} \leq 330^{\circ} ; \zeta^{(17 \mathrm{c})}\left(q_{2}\right): 34^{\circ} \leq q_{2} \leq$ $7^{\circ} ; \zeta^{(10)}\left(q_{3}\right):-20^{\circ} \leq q_{3} \leq 20^{\circ} ;$ and $\zeta^{(18)}\left(q_{2}\right): 0^{\circ} \leq q_{2} \leq 30^{\circ}$ and shown in Figure 5.

The boundary to the first void (void 1 ) is characterized by $\zeta^{(15)}\left(q_{1}\right): 0^{\circ} \leq q_{1} \leq 330^{\circ} ; \zeta^{(9)}\left(q_{3}\right):-17^{\circ} \leq q_{3} \leq 0^{\circ}$; and $\zeta^{(17 b)}\left(q_{2}\right):-\pi \leq q_{2} \leq-175^{\circ}$.

The boundary to the second void (void 2) is characterized by $\xi^{(5)}\left(q_{1}\right): 276^{\circ} \leq q_{1} \leq 283^{\circ} ; \xi^{(17 a)}\left(q_{2}\right):-154^{\circ} \leq q_{2} \leq$ $-70^{\circ} ; \xi^{(8)}\left(q_{1}\right): 293^{\circ} \leq q_{1} \leq 312^{\circ} ;$ and $\xi^{(11)}\left(q_{2}\right):-142^{\circ} \leq q_{2}$ $\leq-30^{\circ}$. All boundary curves are shown in Figure 5 . 


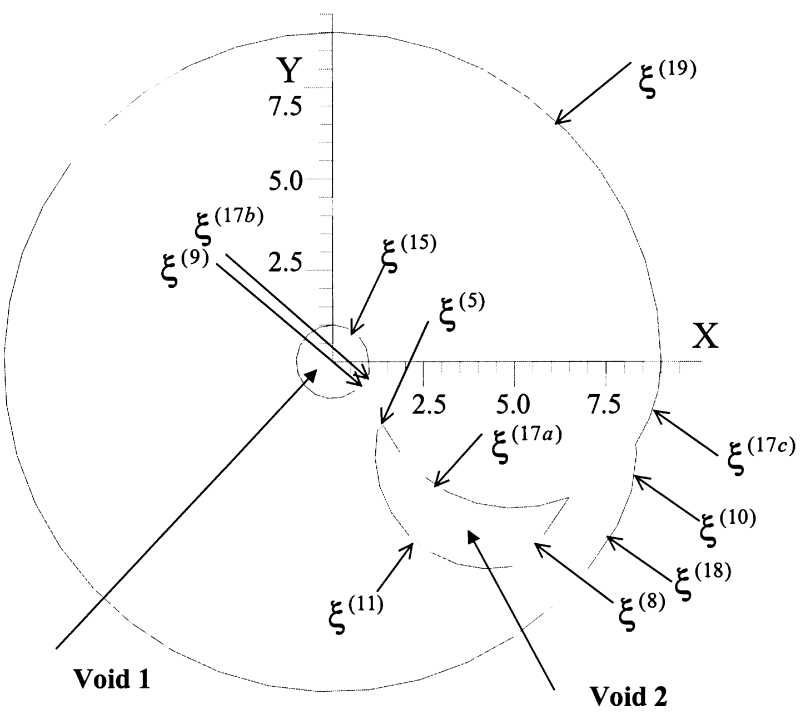

Figure 5 Identification of voids and exterior boundary

\section{A four-parameter sweep}

Consider the sweep of the curve $\mathbf{c}\left(q_{4}\right):-153^{\circ} \leq q_{4} \leq 128^{\circ}$, shown in Figure 6. The curve will first be swept about the axis $Z_{1}$ with an angle $q_{3}: 0 \leq q_{3} \leq 330^{\circ}$ and the resulting shape will be swept along the cylinder $\mathbf{s}\left(q_{1}, q_{2}\right)$ with radius $R$ $=13$, where $-4.5 \leq q_{2} \leq 0$ and $0 \leq q_{1} \leq 270^{\circ}$. Note that the sweep of geometrical entities along surfaces is extensively utilized in $\mathrm{NC}$ verification and robot workspace analysis.

The sweep equation is computed as:

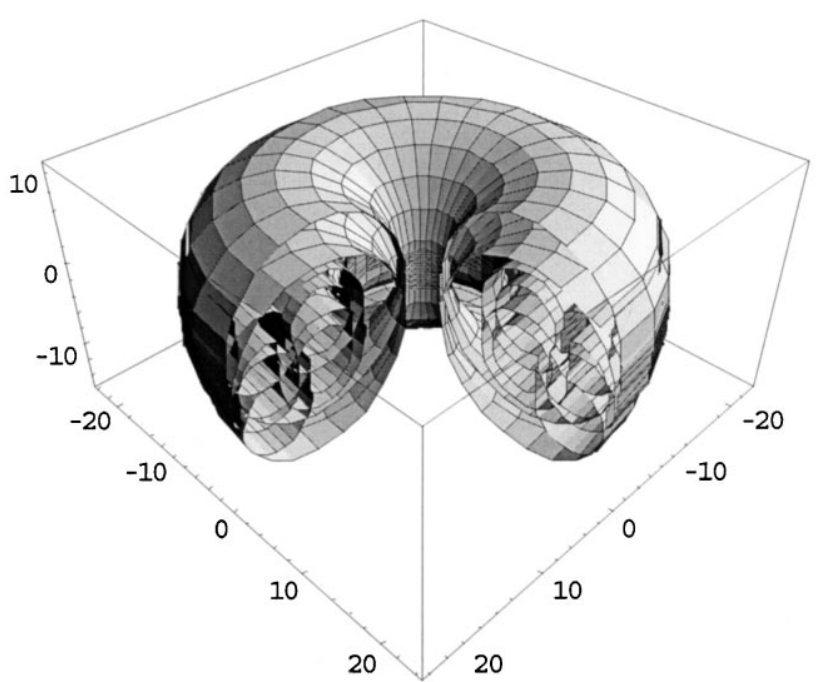

Figure 7 A view of the swept volume depicting hyperentities (note that hypersurfaces at $q_{1}^{\mathrm{L}}$ and $q_{1}^{\mathrm{U}}$ are not shown, in order to show the maximum number of internal surfaces)

$\mathbf{p}^{(23)}=\left\{q_{1}^{\mathrm{L}}, q_{2}^{\mathrm{U}}\right\}, \mathbf{p}^{(24)}=\left\{q_{1}^{\mathrm{L}}, q_{2}^{\mathrm{L}}\right\}, \mathbf{p}^{(25)}=\left\{q_{1}^{\mathrm{L}}, q_{4}^{\mathrm{L}}\right\}, \mathbf{p}^{(26)}=$ $\left\{q_{1}^{\mathrm{L}}, q_{4}^{\mathrm{U}}\right\}, \quad \mathbf{p}^{(27)}=\left\{q_{1}^{\mathrm{U}}, q_{3}^{\mathrm{L}}\right\}, \quad \mathbf{p}^{(28)}=\left\{q_{1}^{\mathrm{U}}, q_{3}^{\mathrm{U}}\right\}, \quad \mathbf{p}^{(29)}=$ $\left\{q_{1}^{\mathrm{U}}, q_{2}^{\mathrm{U}}\right\}, \quad \mathbf{p}^{(30)}=\left\{q_{1}^{\mathrm{U}}, q_{2}^{\mathrm{L}}\right\}, \quad \mathbf{p}^{(31)}=\left\{q_{1}^{\mathrm{U}}, q_{4}^{\mathrm{L}}\right\}, \quad \mathbf{p}^{(32)}=$ $\left\{q_{1}^{\mathrm{U}}, q_{4}^{\mathrm{U}}\right\}$.

Substituting each singularity set into eqn (35) yields parametric equations of hyperentities in $\mathcal{R}^{3}$ shown in Figure 7. Figure 8 is a cross-section (a slice) of the swept volume depicting the trace of each hypersurface. It is required to determine all boundaries to this set. Note that the $Z$-axis is the axis of the cylindrical surface.

$$
\Phi(\mathbf{q})=\left[\begin{array}{c}
-4 \sin q_{1} \cos q_{3} \cos q_{4}+4 \sin q_{1} \sin q_{3} \sin q_{4}-6.6 \sin q_{1} \cos q_{3}-13 \sin q_{1} \\
4 \cos q_{1} \cos q_{3} \cos q_{4}-4 \cos q_{1} \sin q_{3} \sin q_{4}+6.6 \cos q_{1} \cos q_{3}+13 \cos q_{1} \\
4 \sin q_{3} \cos q_{4}+4 \cos q_{3} \sin q_{4}+6.6 \sin q_{3}+q_{2}
\end{array}\right]
$$

where $\mathbf{q}=\left[\begin{array}{llll}q_{1} & q_{2} & q_{3} & q_{4}\end{array}\right]^{\mathrm{T}}$. Jacobian rank-deficiency criteria yield the following singular sets, where the superscripts $\mathrm{U}$ and $\mathrm{L}$ indicate upper and lower limits, respectively. $\quad \mathbf{p}^{(1)}=\left\{q_{2}^{\mathrm{U}}, q_{4}^{\mathrm{L}}\right\}, \quad \mathbf{p}^{(2)}=\left\{q_{2}^{\mathrm{U}}, q_{4}^{\mathrm{L}}\right\}, \quad \mathbf{p}^{(3)}=$ $\left\{q_{2}^{\mathrm{U}}, q_{4}=0\right\}, \quad \mathbf{p}^{(4)}=\left\{q_{2}^{\mathrm{U}}, q_{3}^{\mathrm{L}}\right\}, \quad \mathbf{p}^{(5)}=\left\{q_{2}^{\mathrm{U}}, q_{3}^{\mathrm{U}}\right\}$, $\mathbf{p}^{(6)}=\left\{q_{2}^{\mathrm{L}}, q_{4}^{\mathrm{U}}\right\}, \quad \mathbf{p}^{(7)}=\left\{q_{2}^{\mathrm{L}}, q_{4}^{\mathrm{L}}\right\}, \quad \mathbf{p}^{(8)}=\left\{q_{2}^{\mathrm{L}}, q_{4}=0\right\}$, $\mathbf{p}^{(9)}=\left\{q_{2}^{\mathrm{L}}, q_{3}^{\mathrm{L}}\right\}, \quad \mathbf{p}^{(10)}=\left\{q_{2}^{\mathrm{L}}, q_{3}^{\mathrm{U}}\right\}, \quad \mathbf{p}^{(11)}=\left\{q_{3}=\pi, q_{4}=0\right\}$, $\mathbf{p}^{(12)}=\left\{q_{3}=143.3^{\circ}, q_{4}^{\mathrm{U}}\right\}, \mathbf{p}^{(13)}=\left\{q_{3}=209.5^{\circ}, q_{4}^{\mathrm{L}}\right\}, \mathbf{p}^{(14)}=$ $\left\{q_{3}^{\mathrm{U}}, q_{4}^{\mathrm{L}}\right\}, \quad \mathbf{p}^{(15)}=\left\{q_{3}^{\mathrm{L}}, q_{4}^{\mathrm{L}}\right\}, \quad \mathbf{p}^{(16)}=\left\{q_{3}=30^{\circ}, q_{4}^{\mathrm{L}}\right\}, \quad \mathbf{p}^{(17)}=$ $\left\{q_{3}^{\mathrm{L}}, q_{4}^{\mathrm{U}}\right\}, \quad \mathbf{p}^{(18)}=\left\{q_{3}^{\mathrm{U}}, q_{4}^{\mathrm{U}}\right\}, \quad \mathbf{p}^{(19)}=\left\{q_{3}^{\mathrm{U}}, q_{4}=30^{\circ}\right\}$, $\mathbf{p}^{(20)}=\left\{q_{3}^{\mathrm{L}}, q_{4}=0\right\}, \quad \mathbf{p}^{(21)}=\left\{q_{1}^{\mathrm{L}}, q_{3}^{\mathrm{L}}\right\}, \quad \mathbf{p}^{(22)}=\left\{q_{1}^{\mathrm{L}}, q_{3}^{\mathrm{U}}\right\}$,

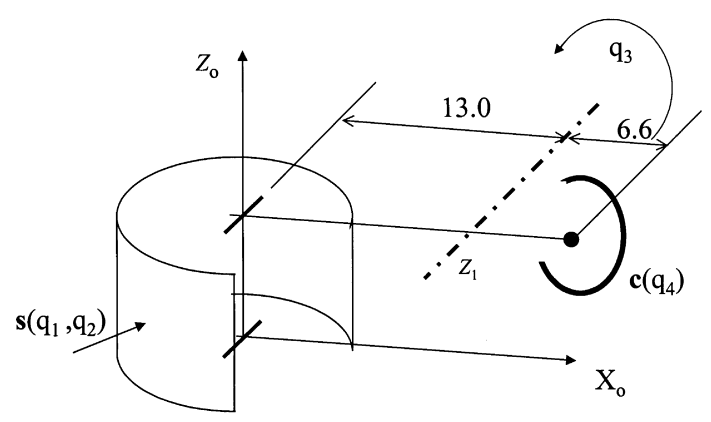

Figure 6 Four-parameter sweep
In order to demonstrate the formulation for the fourparameter sweep, we shall trace the numerical algorithm (Appendix A) for two points on the same hypersurface. One of the points will identify a surface patch as a boundary but not the other.

For the hypersurface $\xi^{(7)}(\mathbf{u}), \mathbf{u}^{(7)}=-\left[\begin{array}{ll}q_{1} & q_{3}\end{array}\right]^{\mathrm{T}}$, and $\mathbf{p}^{(7)}=\left[\begin{array}{ll}q_{2}^{\mathrm{L}} & q_{4}^{\mathrm{L}}\end{array}\right]^{\mathrm{T}}$, the $(4 \times 3)$ matrix. At the point $\mathbf{q}_{0}=\left[\begin{array}{llll}5^{\circ} & q_{2}^{\mathrm{L}} & 300^{\circ} & q_{4}^{\mathrm{L}}\end{array}\right]^{\mathrm{T}}$ shown in Figure 9:

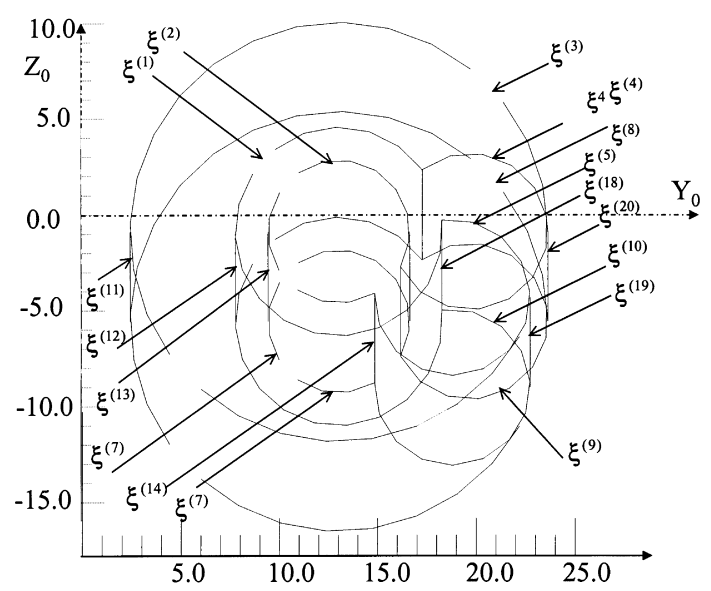

Figure 8 A slice of the swept volume depicting the trace of each hyperentity 


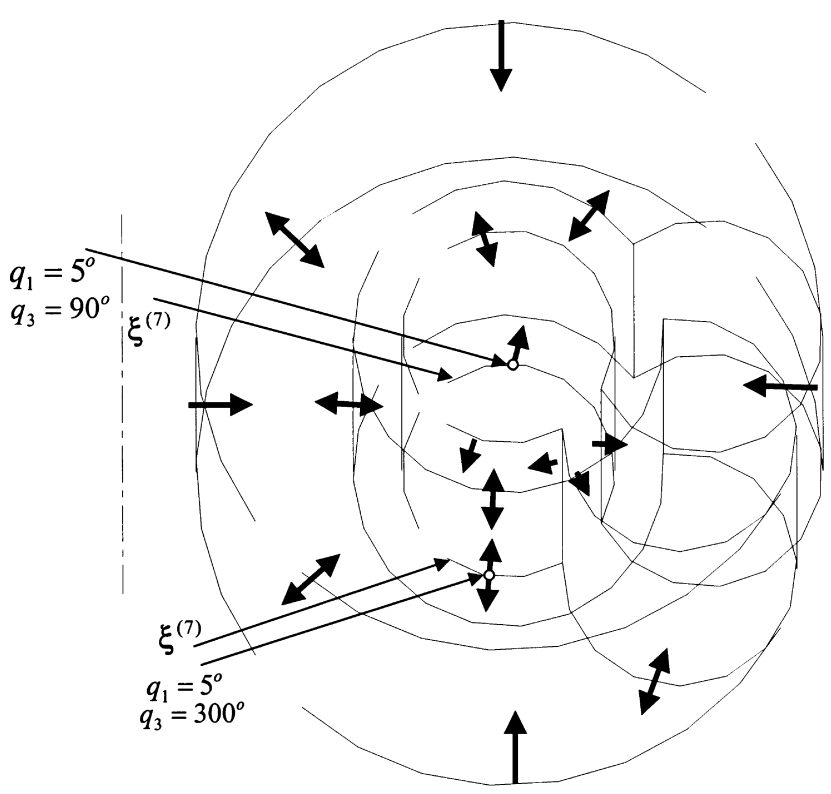

Figure 9 A cross-section of the swept volume depicting the trace of hyperentities and admissable normal directions

$$
\left[\Phi_{\mathrm{q}} \mathbf{q}_{\mathrm{s}}\right]^{\mathrm{T}}=\left[\begin{array}{ccc}
-8.1923 & -0.7168 & 0 \\
0 & 0 & 0 \\
-0.5104 & 5.8345 & -0.0905 \\
0 & 0 & 0
\end{array}\right]
$$

The nullspace of $\left[\Phi_{\mathrm{q}} \mathbf{q}_{\mathrm{s}}\right]^{\mathrm{T}}$ is $\mathbf{n}_{0}=$ $\left[\begin{array}{lll}-0.0013 & 0.01539 & 0.9998\end{array}\right]^{\mathrm{T}}$. The computation of:

$$
\mathbf{q}_{\mathrm{s}}^{\mathrm{T}}\left[\mathbf{n}_{0}^{\mathrm{T}} \Phi\right]_{\mathrm{qq}} \mathbf{q}_{\mathrm{s}}=\left[\begin{array}{cccc}
-0.0807 & 0 & 0 & 0 \\
0 & 0 & 9.6901 & 0 \\
0 & 0 & 0 & 0
\end{array}\right]
$$

and

$$
\sum_{i=1}^{n+m} \frac{\mathrm{d} \Phi}{\mathrm{d} q_{1}}\left[q_{1}\right]_{\mathrm{ss}}=\left[\begin{array}{cccc}
-0.0807 & 0 & 0 & 0 \\
0 & 2.2477 & 0 & 0 \\
0 & 0 & 9.6899 & 0 \\
0 & 0 & 0 & -8.3007
\end{array}\right]
$$

For the hypersurface, since:

$$
\mathbf{E}=\left[\begin{array}{lll}
1 & 0 & 0 \\
0 & 0 & 1
\end{array}\right]
$$

the determinant of $\operatorname{Det}\left[\mathbf{E} \xi_{\mathrm{u}}\right]=0.705$, then:

$$
\mathbf{B}=\left[\mathbf{E} \xi_{\mathrm{u}}\right]^{-1} \mathbf{E}=\left[\begin{array}{ccc}
-0.0775 & 0 & 0.4372 \\
0 & 0 & -18.2875
\end{array}\right]
$$

The eigenvalues of $\mathbf{Q}^{*}$ are $\{-8.3001,2.2477,0,0\}$. Since this is an indefinite form, this surface patch is not a boundary, i.e. that region of the hypersurface. Two arrowheads are shown in Figure 9 to denote that movement is admitted along both directions.

However, for the same hypersurface at the point $\mathbf{q}_{0}=\left[\begin{array}{llll}5^{\circ} & q_{2}^{\mathrm{L}} & 90^{\circ} & q_{4}^{\mathrm{L}}\end{array}\right]^{\mathrm{T}}, \quad$ the normal is $\mathbf{n}_{0}=\left[\begin{array}{lll}0.0447 & -0.5114 & -0.8582\end{array}\right]^{\mathrm{T}}$, and the resulting eigenvalues are $\{-8.3037,-1.9305,0,0\}$, which is a negative semi-definite form. Therefore, $\sigma=n^{\mathrm{T}} \Phi_{q_{2}} \delta q_{2}=-0.707$ is calculated where at the lower limit $q_{2}^{\mathrm{L}}, \delta q_{2}=+1$. This indicates admissible motion only towards the opposite normal direction, i.e. a boundary has been identified (Figure 9).

Boundary hypersurface regions are identified by applying the criteria. The void is identified, as presented in Table 1 and shown in Figure 10 (only one cross-section is shown).

The exterior boundary is identified and entered into Table 2 and shown in Figure 10. The complete swept volume after removing those hypereneities that admit motion, is shown in Figure 11. Note that exact closed form equations are now identified for each surface patch enveloping the volume including voids.

\section{CONCLUSIONS}

A mathematical formulation for identifying all boundaries to the swept volume, including voids, was presented. The concept of design using multiple sweeps was presented elsewhere and is used here to generate all hyperentities associated with the sweep.

Using a normal acceleration function adpated from kinematics, the movement of a point on a hypersurface is addressed. Criteria are derived regarding the point's

Table 1 The void in the four-parameter sweep

\begin{tabular}{llll}
\hline Hypersurface & Parameters & Singular set & Limits \\
\hline$\xi^{(3)}(\mathbf{u})$ & $\mathbf{u}^{(3)}=\left[\begin{array}{ll}q_{1} & q_{3}\end{array}\right]^{\mathrm{T}}$ & $\mathbf{p}^{(3)}$ & $0 \leq q_{1} \leq 270^{\circ}$ and $0 \leq q_{3} \leq \pi$ \\
$\xi^{(8)}(\mathbf{u})$ & $\mathbf{u}^{(8)}=\left[\begin{array}{ll}q_{1} & q_{3}\end{array}\right]^{\mathrm{T}}$ & $\mathbf{p}^{(8)}$ & $0 \leq q_{1} \leq 270^{\circ}$ and $0 \leq q_{3} \leq 330^{\circ}$ \\
$\xi^{(11)}(\mathbf{u})$ & $\mathbf{u}^{(11)}=\left[\begin{array}{ll}q_{1} & q_{2}\end{array}\right]^{\mathrm{T}}$ & $\mathbf{p}^{(11)}$ & $0 \leq q_{1} \leq 270^{\circ}$ and $-4.5 \leq q_{2} \leq 0$ \\
$\xi^{(20)}(\mathbf{u})$ & $\mathbf{u}^{(20)}=\left[\begin{array}{ll}q_{1} & q_{2}\end{array}\right]^{\mathrm{T}}$ & $\mathbf{p}^{(20)}$ & $0 \leq q_{1} \leq 270^{\circ}$ and $-4.5 \leq q_{2} \leq 0$ \\
$\xi^{(9)}(\mathbf{u})$ & $\mathbf{u}^{(9)}=\left[\begin{array}{ll}q_{1} & q_{4}\end{array}\right]^{\mathrm{T}}$ & $\mathbf{p}^{(9)}$ & $0 \leq q_{1} \leq 270^{\circ}$ and $-153^{\circ} \leq q_{4} \leq 128^{\circ}$ \\
$\xi^{(10)}(\mathbf{u})$ & $\mathbf{p}^{(10)}=\left[\begin{array}{ll}q_{1} & q_{4}\end{array}\right]^{\mathrm{T}}$ & $\mathbf{p}^{(10)}$ & $0 \leq q_{1} \leq 270^{\circ}$ and $-153^{\circ} \leq q_{4} \leq 128^{\circ}$ \\
\hline
\end{tabular}

Table 2 The void in the four-parameter sweep

\begin{tabular}{llll}
\hline Hypersurface & Parameters & Singular set & Limits \\
\hline$\xi^{(7)}(\mathbf{u})$ & $\mathbf{u}^{(7)}=\left[\begin{array}{ll}q_{1} & q_{3}\end{array}\right]^{\mathrm{T}}$ & $\mathbf{p}^{(7)}$ & $0 \leq q_{1} \leq 270^{\circ}$ and $60.4^{\circ} \leq q_{3} \leq 169.9^{\circ}$ \\
$\xi^{(2)}(\mathbf{u})$ & $\mathbf{u}^{(2)}=\left[\begin{array}{ll}q_{1} & q_{3}\end{array}\right]^{\mathrm{T}}$ & $\mathbf{p}^{(2)}$ & $0 \leq q_{1} \leq 270^{\circ}$ and $249^{\circ} \leq q_{3} \leq 330^{\circ}$ \\
$\xi^{(15)}(\mathbf{u})$ & $\mathbf{u}^{(15)}=\left[\begin{array}{ll}q_{1} & q_{2}\end{array}\right]^{\mathrm{T}}$ & $\mathbf{p}^{(15)}$ & $0 \leq q_{1} \leq 270^{\circ}$ and $-2.28 \leq q_{2} \leq-0.93$ \\
$\xi^{(14)}(\mathbf{u})$ & $\mathbf{u}^{(14)}=\left[\begin{array}{ll}q_{1} & q_{2}\end{array}\right]^{\mathrm{T}}$ & $\mathbf{p}^{(14)}$ & $0 \leq q_{1} \leq 270^{\circ}$ and $-1.8 \leq q_{2} \leq 0$ \\
$\xi^{(1)}(\mathbf{u})$ & $\mathbf{u}^{(1)}=\left[\begin{array}{ll}q_{1} & q_{3}\end{array}\right]^{\mathrm{T}}$ & $\mathbf{p}^{(1)}$ & $0 \leq q_{1} \leq 270^{\circ}$ and $257.8^{\circ} \leq q_{3} \leq 270^{\circ}$ \\
\hline
\end{tabular}




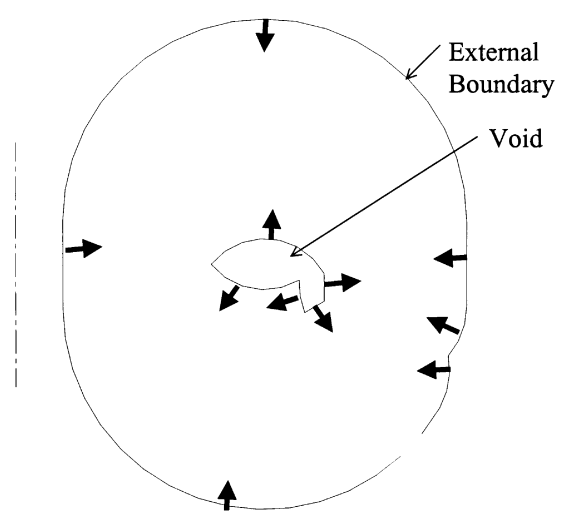

Figure 10 A cross-section (a longitudinal slice) due to $q_{1}=\pi / 2$

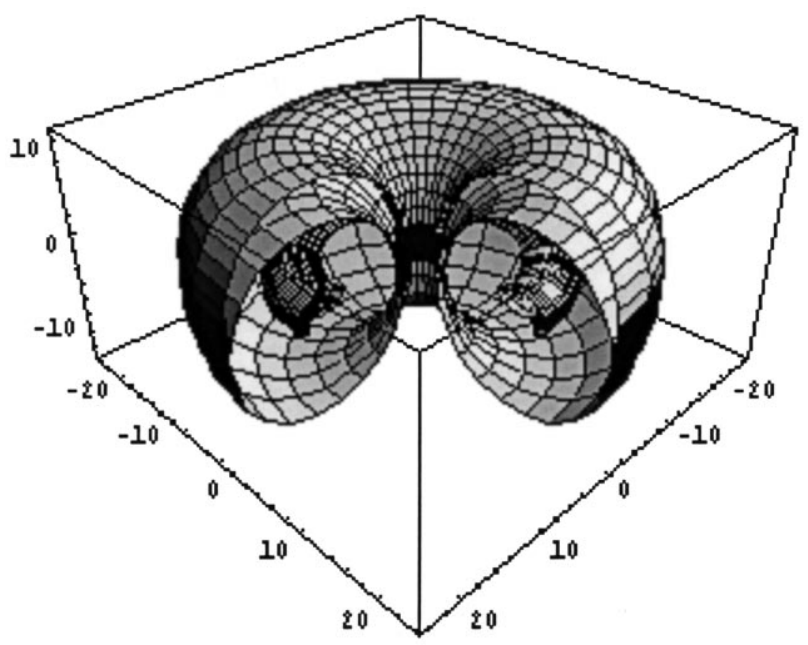

Figure 11 A cross-section of the swept volume depicting all bounding surface patches movement into enclosures that do not belong to the swept volume and hence identifying a boundary. The formulation was shown to be exact, as it is completely analytical without any approximate assumptions.

It was shown that a hyperentity exhibits only onedirectional movement in the opposite direction to the void if the hyperentity is on the boundary of that void or on the boundary of an external entity. Criteria based on definiteness properties of a quadratic form derived from the acceleration analysis were used to define the boundary. It was also shown that, at the edge of a hyperentity (a parameter at its limit), an additional criterion is necessary to determine whether a surface patch on that entity is on the boundary. The additional criterion is needed because of the loss of information in the quadratic term due to zero enforced velocity on the hypersurface.

Illustrative examples are given to validate the presented theory. It is evident that, since all boundaries were determined, and because a closed-form expression exists for each boundary, an exact determination of the swept volume including voids is obtained. While it is only an experimental code, it was shown that this method is capable of handling complex sweeps.

\section{ACKNOWLEDGEMENTS}

The authors gratefully acknowledge the support of the Department of Defense under grant number DAAE07-94C-R094 and the Center for Computer-Aided Design.

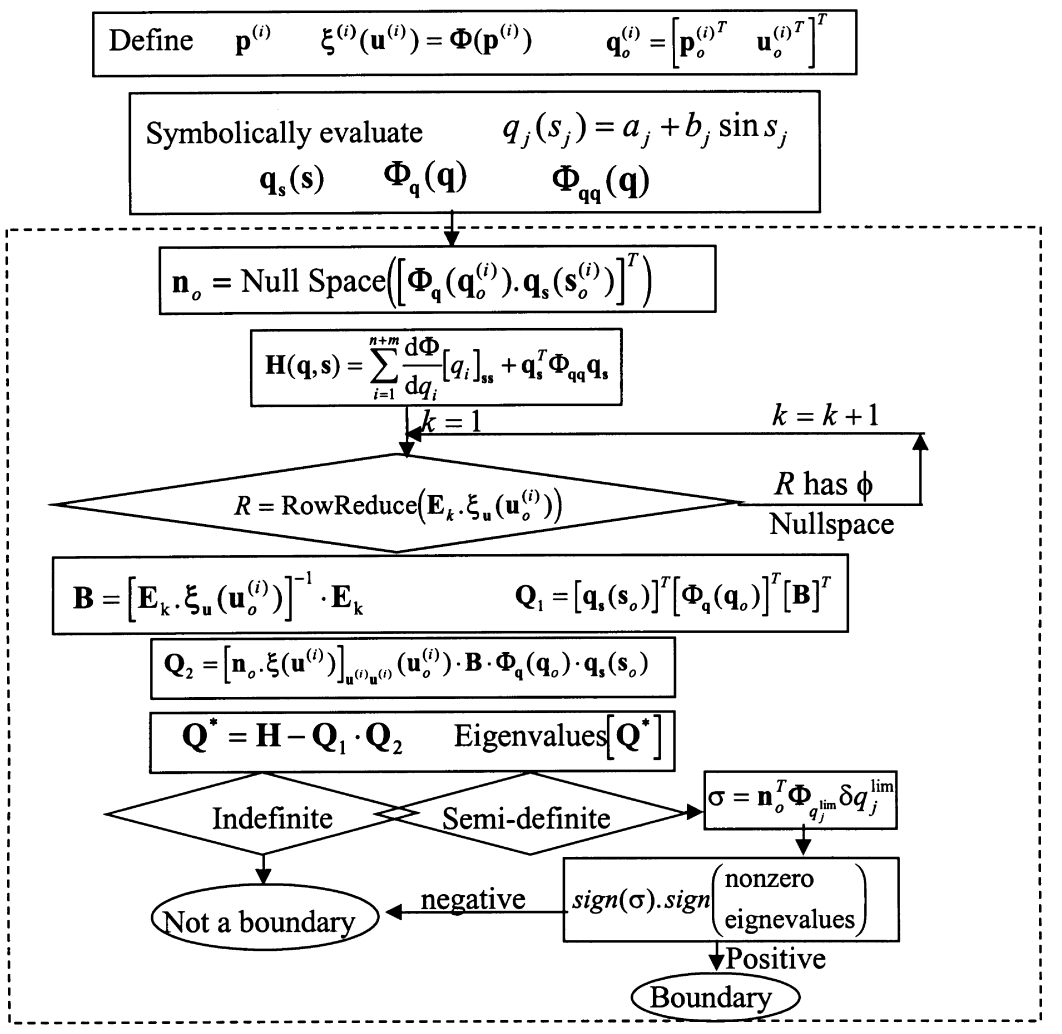

Figure 12 Boundary identification algorithm for each hyperentity 


\section{APPENDIX ALGORITHM FOR DETERMINING A BOUNDARY}

The experimental code was written using Mathematica ${ }^{\circledR}$ such that the derivatives are performed symbolically. The operations contained within the dotted rectangle indicate that they are computed for each point on a hyperentity (surface patch). The superscript (i) denotes a hyperentity defined by the singular set, $\mathbf{p}^{(i)}$, and its corresponding vector function, $\xi^{(i)}$. If the form is semi-definite, the sign of $\sigma$ is multiplied with that of the nonzero eigenvalues. If both are of the same sign, then it yields a positive sign indicating a boundary. The algorithm is shown in Figure 12.

\section{REFERENCES}

1. Abdel-Malek, K. and Yeh, H. J., Geometric representation of the swept volume using Jacobian rank deficiency conditions. ComputerAided Design, 1997, 29(6), 457-468.

2. Abrams, S. and Allen, P. K. Swept volumes and their use in viwpoint computation in robot work-cells. In Proceedings of the IEEE International Symposium on Assembly and Task Planning [1995] pp. 188193.

3. Ahn, J. W., Kim, M. S. and Lim, S. B., Approximate general sweep boundary of a 2D curved object. Computer Vision, Graphics and Image Processing, 1993, 55(2), 98-128.

4. Blackmore, D. and Leu, M. C., Analysis of swept volume via lie groups and differential equations. The International Journal of Robotics Research, 1992, 11(6), 516-537.

5. Blackmore, D., Jiang, H. and Leu, M. C. Modeling of swept solids using sweep differential techniques. In Proceedings of the 4th IFIP WG5.2 Workshop Geometric Modeling in Computer-Aided Design, Rensselaerville, NU [1992].

6. Blackmore, D., Leu, M. C. and Shih, F., Analysis and modeling of deformed swept volumes. Computer-Aided Design, 1994, 26(4), 315326.

7. Bronsvoort, W., Van Nieuwenhuizen, P. and Post, F., Display of profiled sweep objects. The Visual Computer, 1989, 5(3), 147-157.

8. Coquillart, S. A., Control point based sweeping technique. IEEE Computer Graphics and Applications, 1987, 7(11), 36-44.

9. Farin, G. (1993). Curves and surfaces for computer-aided geometric design. Academic Press: San Diego, CA.

10. Fridshal, R., Duncan, D., Cheng, K. D. and Zucker, W. Numerical control part program verification system. In Proceedings CAD/CAM Technology for Mechanical Engineering. MIT: Cambridge, MA, 1982, pp. 236-254.

11. Ganter, M. A., Stori, D. W. and Ensz, M. T., On algebraic methods for implicit swept solids with finite extent. ASME Advances in Design Automation, 1993, 65(2), 389-396.

12. Hu, Z. J. and Ling, Z. K. Generating swept volumes with instantaneous screw aces. In Proceedings of the 1994 ASME Design Technical Conference, Part 1, Minneapolis, MN [1994] Vol. 70(1), pp. 7-14.

13. Hu, Z. J. and Ling, Z. K., Swept volumes generated by the natural quadric surfaces. Computers and Graphics, 1996, 20(2), 263-274.

14. Jerard, R. B. and Drysdale, R. L., Geometric simulation of numerical control machinery. ASME Computers Engineer, 1988, 2, 129-136.

15. Kieffer, J. and Litvin, F. L., Swept volume determination and interference detection for moving 3-D solids. Transactions of the ASME, Journal of Mechanical Design, 1990, 113, 456-463.
16. Klok, F., Two moving coordinate frames for sweeping along a 3D trajectory. Computer-Aided Geometric Design, 1986, 3, 217-229.

17. Leu, M. C., Park, S. H. and Wang, K. K., Geometric representation of translational swept volumes and its applications. ASME Journal of Engineering for Industry, 1986, 108, 113-119.

18. Martin, R. R. and Stephenson, P. C., Sweeping of three-dimensional objects. Computer-Aided Design, 1990, 22(4), 223-234.

19. Oliver, J. and Goodman, E., Direct dimensional NC verification. Computer-Aided Design, 1990, 22, 3-9.

20. Schroeder, W. J., Lorensen, E. E. and Linthicum, S. Implicit modeling of swept surfaces and volumes. In Proceedings of the IEEE Visualization Conference, Los Alamitos, CA [1994] pp. 40-45.

21. Voelker, H. B. and Hunt, W. A. The role of solid modeling in machining process modeling and NC verification. SAE Technical Paper, no. 810195 [1985].

22. Wang, W. P. and Wang, K. K., Geometric modeling for swept volume of moving solids. IEEE Computer Graphics and Applications, 1986, 6(12), 8-17.

23. Weld, J. D. and Leu, M. C., Goemetric representation of swept volumes with application to polyhedral objects. The International Journal of Robotics Research, 1990, 9(5), 105-117.

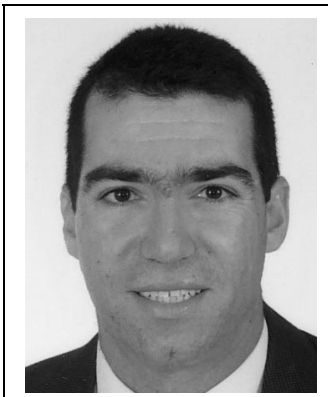

Karim Abdel-Malek is an Assistant Professor of Mechanical Engineering at the University of Iowa, Iowa City. He received his $M S$ and PhD degrees in Mechanical Engineering from the University of Pennsylvania and his BS in Mechanical Engineering from the University of Jordan in Amman. He worked for the manufacturing industry for a number of years before joining the faculty at Iowa. His research interests are in

$C A D / C A M$, robotics and kinematics.

Harn-Jou Yeh is a senior research engineer at Microtek Inc., in Taiwan. He received his $M S$ and PhD degrees from the University of Iowa in Mechanical Engineering. He received his BS in Mechanical Engineering from the National Taiwan University. He worked as a senior researcher at the Center for Computer-Aided Design (CCAD) for two years before accepting a position at Microtek. Dr. Yeh's research interests are in kinematics, CAD and robotics.

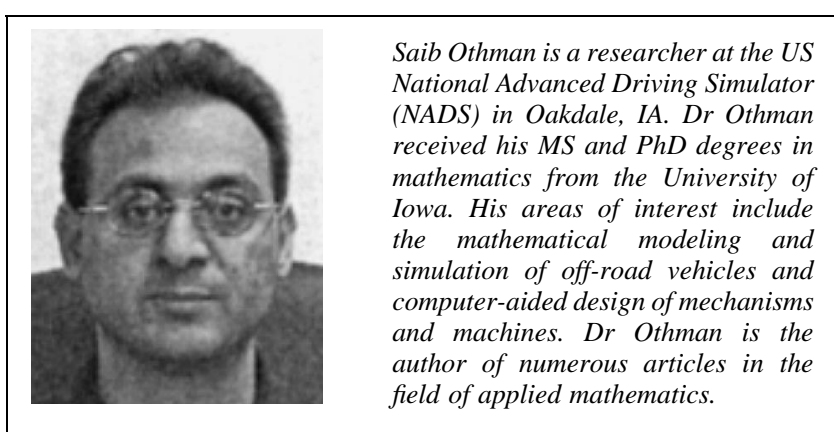

